\title{
What's in a Number? Risk Thresholds in Different Statin Guidelines
}

\author{
Christopher J. Wong, $M D^{7}$ and Lisa Inouye, $M D^{2}$
}

'Division of General Internal Medicine, Department of Medicine, University of Washington, Seattle, WA, USA; ${ }^{2}$ Division of General Internal Medicine, Department of Medicine, University of Washington, Boise Veterans Affairs Medical Center, Boise, ID, USA.

$\mathrm{J}$ Gen Intern Med 32(10):1071-3

DOI: $10.1007 / \mathrm{s} 11606-017-4114-\mathrm{y}$

() Society of General Internal Medicine 2017

$\mathrm{W}$ hen exactly should we recommend statin therapy to our patients for primary prevention? Clinical practice guidelines published since the 2013 American College of Cardiology/American Heart Association (ACC/AHA) guidelines recommend quite a range of different treatment thresholds. Imagine the veteran who starts in a private practice, is recommended a $7.5 \%$ 10-year risk threshold to start statin therapy, then transfers to the VA system, where the threshold changes to $12 \%$, and then moves to Canada, where the threshold goes back to $10 \%$.

While the itinerant patient example above may seem absurd, it highlights that there is more than just evidence-based medicine at play: guideline creation is as much art as it is cold hard evidence. At first glance, the evidence is that statins reduce cardiovascular outcomes in primary prevention across a wide range of baseline risk. ${ }^{1}$ On closer inspection, the art comes when we have to decide when and for whom to apply this risk reduction.

The modern guidelines shown in Table 1 have some common features, as they attempt to transform evidence into primary prevention recommendations. The majority of guidelines recommend statin therapy based on risk rather than treating to LDL targets. They all advocate the use of risk thresholds divided into discrete categories based on the 10year risk of cardiovascular events as estimated by various risk calculators. Some of the guidelines use a "low/moderate/highrisk" model: a low-risk category for which no statin therapy is recommended, an intermediate category with either a weakerstrength recommendation (e.g. "consider" treating) or a recommendation for a lower drug dose, and a high-risk category for which treatment is more strongly recommended. In contrast, other guidelines use a dichotomous "low/high-risk" model ("do not treat/treat").

If we look at the thresholds at which these guidelines recommend statin therapy for primary prevention, we find that

Received March 20, 2016

Revised May 19, 2017

Accepted June 14, 2017

Published online June 29, 2017 they vary from $7.5 \%$ to $12 \%$. So why have these guidelines come up with different numbers? It is not because there are new clinical trials that have evaluated the outcomes of treatment based on different risk thresholds. The different clinical practice guidelines, by and large, were based on the same pool of studies: primary prevention trials of statin therapy that typically enrolled subjects based on cardiovascular risk factors and randomized them to a fixed dose of statin versus placebo. Instead, the reason for the difference in threshold recommendations is more likely that the concept of providing appropriate treatment based on risk, however sound, is challenging to translate into thresholds at which the benefits sufficiently outweigh the risks. In particular, the benefits and risks are hard to quantify. How much cardiovascular disease is worth preventing, and how many unnecessary or harmful treatments are we willing to accept to achieve a given level of prevention? The randomized controlled trials cannot answer these questions for us.

We do not yet have a consensus framework on how best to set a treatment threshold. Cost-benefit analysis is a common method, and although it has since been applied to statin treatment thresholds, ${ }^{9}$ most guidelines did not incorporate this type of analysis in formulating their recommendations. Furthermore, cost-benefit analysis using quality-adjusted life years as an outcome is but one means of balancing benefit and harm, is itself controversial, and does not fully incorporate patient values, ethics, and implementation. Without a commonly agreed-upon framework beyond the dollar values of cost-effectiveness analyses, it is unlikely any guideline committee could come up with a number that everyone would - or should - agree with.

Even if we could agree on how to establish a risk threshold, having treatment boil down to a single estimated number can feel uncomfortable, and for good reason. Is a patient estimated as having a $6 \%$ risk really so different from another who checks in at $8 \%$ ? Furthermore, risk calculators vary widely in their ability to include racially diverse populations and those with other medical conditions. A review of recent data found that risk calculators often overestimate risk in real-world populations. ${ }^{10}$

Still, we commend these clinical guidelines for providing some kind of barometer for statin therapy. Guidelines should not just restate existing data $—$ on some level there must always be value and judgment in a recommendation that simplifies our decision-making. How then should we approach the use of statin therapy in primary prevention with our patients, in the face of so many guidelines, each with distinct recommendations? 


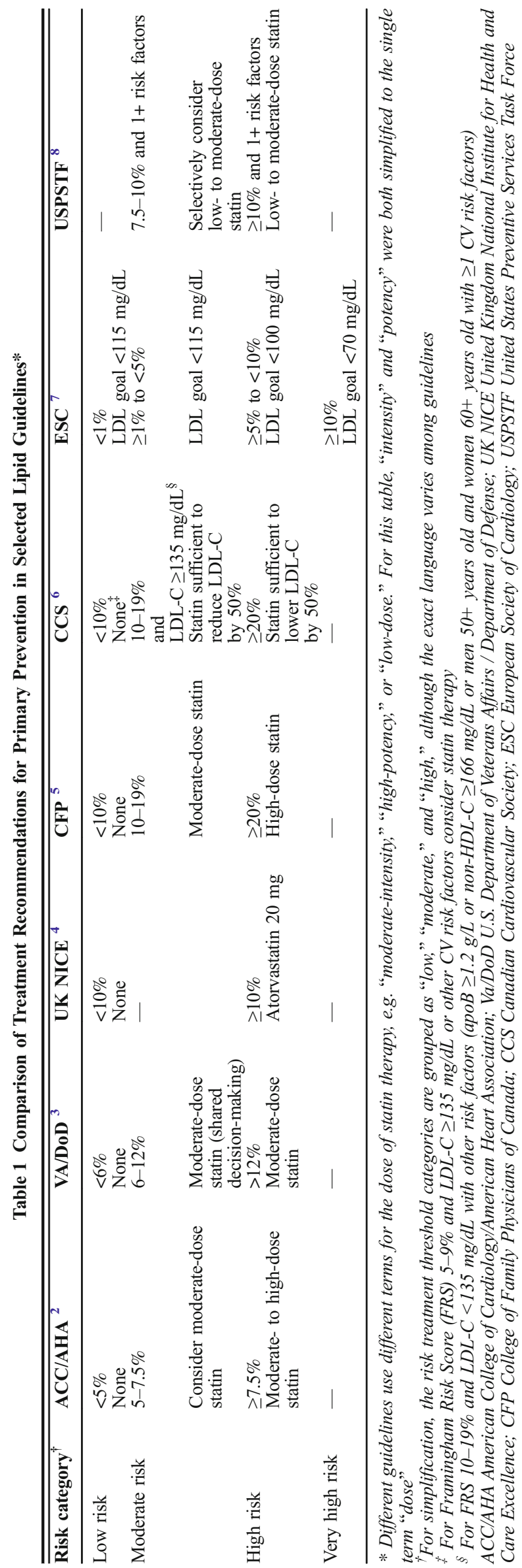

First, we urge physicians not to be overly reliant on a single guideline's threshold, be it $7.5 \%, 12 \%$, or whatever number and risk stratification scheme the next guideline committee comes up with - it's an impossible task. We should be upfront with our patients that there are recommendations from prominent societies whose conclusions differ, and that they generally recommend considering treatment at a 10 -year risk above about $10 \%$, give or take a few percentage points. We should explain that these risk calculators provide only an estimate and have not been studied in all populations. Because the use of risk thresholds alone would lead to nearly all patients being recommended treatment once they reach a certain age, it is important to discuss the USPSTF's criterion that patients also have a cardiovascular risk factor.

Second, we should help patients understand the difference between individual and population risk. We should avoid the practice of telling patients that by taking a statin, they will lower their risk from, say, $15 \%$ to $10 \%$, or $3 \%$ to $2 \%$. While conceptually easy to understand, it is not entirely accurate: this reduction applies to a population of patients with the same profile, whereas we cannot know whether an individual patient will receive benefit, harm, both, or neither. Decision aids such as that available online from the Mayo $\mathrm{Clinic}^{11}$ appropriately illustrate this principle.

Third, we should spend time with our patients qualitatively exploring their values. Each of these guidelines appropriately recommends shared decision-making, but how do we accomplish the task of making an individual decision on the basis of a population risk tool and in the face of varying guidelines?

Consider this: the lifetime risk of cardiovascular disease tends to be quite high-30-60\% in the United States, ${ }^{12}$ well exceeding the treatment thresholds of every guideline. So perhaps the better focus of our attention should be on using a framework not for population-based risk thresholds, but instead on an individual level, exploring in depth a patient's values, as nearly every patient could potentially reach a treatment threshold for most guidelines if they lived long enough. How concerned is the patient about cardiovascular disease? Does taking a pill every day to reduce possible risk seem "worth it" to the patient? We must ensure that patients' decisions are consistent with their values and goals. Language is important-we might call statin therapy an "optional" preventive drug. In this way, a statin drug is merely another component of an overall cardiovascular risk reduction strategy that should include exercise, smoking cessation, and control of other risk factors such as hypertension. With preventive drug therapy, it is not easy to weigh the possibility of side effects against the unknown of whether an individual patient will reap the benefit. And yet we must guide our patients through this more difficult discussion, a discussion that is more important than a particular "threshold to treat."

Finally, we should make sure that this choice, to take a drug without knowing for sure if it will actually help, 
remains an individual decision. We should stand up to external forces that may seek to impose a one-size-fitsall statin prescription upon our patients. Furthermore, despite our current era of ever-increasing demands for our time, we should not rush such decisions. Patients may not know how they feel about the concept of chemoprevention, and they may need to go home and think about it. Allowing this time and providing follow-up may ultimately be more important than debating the finer points of a risk threshold.

Corresponding Author: Christopher J. Wong, MD; Division of General Internal Medicine, Department of MedicineUniversity of Washington, Seattle, WA, USA (e-mail: cjwong@uw.edu).

\section{Compliance with Ethical Standards:}

Conflict of Interest: The authors declare no conflicts of interest.

\section{REFERENCES}

1. Taylor F, Huffman MD, Macedo AF, et al. Statins for the primary prevention of cardiovascular disease. Cochrane Database Syst Rev. 2013; 1:CD004816.

2. Stone NJ, Robinson JG, Lichtenstein AH, et al. 2013 ACC/AHA guideline on the treatment of blood cholesterol to reduce atherosclerotic cardiovascular risk in adults: a report of the American College of Cardiology/American Heart Association Task Force on Practice Guidelines. J Am Coll Cardiol. 2014;63(25B):2889-934.
3. Downs JR, O'Malley PG. Management of dyslipidemia for cardiovascular disease risk reduction: synopsis of the 2014 U.S. Department of Veterans Affairs and U.S. Department of Defense clinical practice guideline. Ann Intern Med. 2015;163(4):291-7.

4. NICE guidelines [CG181]. Cardiovascular disease: risk assessment and reduction, including lipid modification. Publication date July 2014 Accessed online at http://www.nice.org.uk/guidance/cg181 February 23, 2016.

5. Allan GM, Lindblad AJ, Comeau A, et al. Simplified lipid guidelines: Prevention and management of cardiovascular disease in primary care. Can Fam Physician. 2015;61(10):857-67.

6. Anderson TJ, Gregoire J, Pearson GJ, et al. 2016 Canadian Cardiovascular Society Guidelines for the Management of Dyslipidemia for the Prevention of Cardiovascular Disease in the Adult. Can $\mathrm{J}$ Cardiol. 2016:1-20.

7. Piepoli MF, Hoes AW, Agewall S, et al. 2016 European Guidelines on cardiovascular disease prevention in clinical practice: The Sixth Joint Task Force of the European Society of Cardiology and Other Societies on Cardiovascular Disease Prevention in Clinical Practice (constituted by representatives of 10 societies and by invited experts) Developed with the special contribution of the European Association for Cardiovascular Prevention \& Rehabilitation (EACPR). Eur Heart J. 2016;37(29):2315-81.

8. US Preventive Services Task Force, Bibbins-Domingo $\mathbf{K}$, Grossman DC, et al. Statin Use for the Primary Prevention of Cardiovascular Disease in Adults: US Preventive Services Task Force Recommendation Statement. JAMA. 2016;316(19):1997-2007.

9. Pandya A, Sy S, Cho S, et al. Cost-effectiveness of 10-year risk thresholds for initiation of statin therapy for primary prevention of cardiovascular disease. JAMA. 2015;314(2):142-50.

10. Cook NR, Ridker PM. Calibration of the pooled cohort equations for atherosclerotic cardiovascular disease: an update. Ann Intern Med. 2016;165(11):786-94.

11. Mayo Clinic Statin Choice Decision Aid. Available at: https:// statindecisionaid.mayoclinic.org/. Accessed May 8, 2017.

12. Berry JD, Dyer A, Cai X, et al. Lifetime risks of cardiovascular disease. N Engl J Med. 2012;366(4):321-9. 